\title{
Hydration effects of moisturizing gel on normal skin: A pilot study
}

\author{
Joanna Xuan Hui Goh ${ }^{1}$, Loh Teng-Hern Tan ${ }^{1,2,3^{*}}$, Han Choi Yew ${ }^{4}$, Priyia Pusparajah ${ }^{5}$, Prithvy Lingham ${ }^{5}$, \\ Chiau Ming Long ${ }^{6}$, Learn-Han Lee ${ }^{2,7^{*}}$, Bey-Hing Goh ${ }^{1,7^{*}}$ \\ 'Biofunctional Molecule Exploratory (BMEX) Research Group, School of Pharmacy, Monash University Malaysia, 47500 \\ Bandar Sunway, Selangor Darul Ehsan, Malaysia. \\ ${ }^{2}$ Novel Bacteria and Drug Discovery (NBDD) Research Group, Microbiome and Bioresource Research Strength, Jeffrey \\ Cheah School of Medicine and Health Sciences, Monash University Malaysia, 47500 Bandar Sunway, Selangor Darul Ehsan, \\ Malaysia. \\ ${ }^{3}$ Institute of Biomedical and Pharmaceutical Sciences, Guangdong University of Technology, Guangzhou 510006, PR China. \\ ${ }^{4}$ Department of Chemistry, Faculty of Science, University Malaya, 50603 Kuala Lumpur \\ ${ }^{5}$ Medical Health and Translational Research Group, Jeffrey Cheah School of Medicine and Health Sciences, Monash Univer- \\ sity Malaysia, Bandar Sunway, Selangor, Malaysia. \\ ${ }^{6}$ School of Pharmacy, KPJ Healthcare University College, Nilai, Negeri Sembilan, Malaysia \\ ${ }^{7}$ Institute of Pharmaceutical Science, University of Veterinary and Animal Science Lahore, Pakistan.
}

\begin{abstract}
Moisturizers are topically applied agents which can increase the moisture content in the skin to maintain skin integrity and youthful appearance. L-brand soothing gel (Tested gel) was developed for skin hydration. The objective of this study is to evaluate the benefits of daily usage of Tested gel amongst healthy young adults. This study also aimed to assess whether storage temperature may affect the efficacy of Tested gel. Changes in moisture content and texture index of the skin where the product was applied over a period of 8 days were assessed using non-invasive devices to measure the skin's bioelectric impedance. Our results indicate that application of Tested gel produces a hydrating effect characterized by a significant increment in skin moisture and smoothness. There was no association found between storage temperature and efficacy of gel. The hydration effect of Tested gel diminishes gradually after application, but the hydration effect is cumulative upon daily usage for healthy young adults.
\end{abstract}

Keywords: Moisturizer; hydration; skin; gel; aloe vera

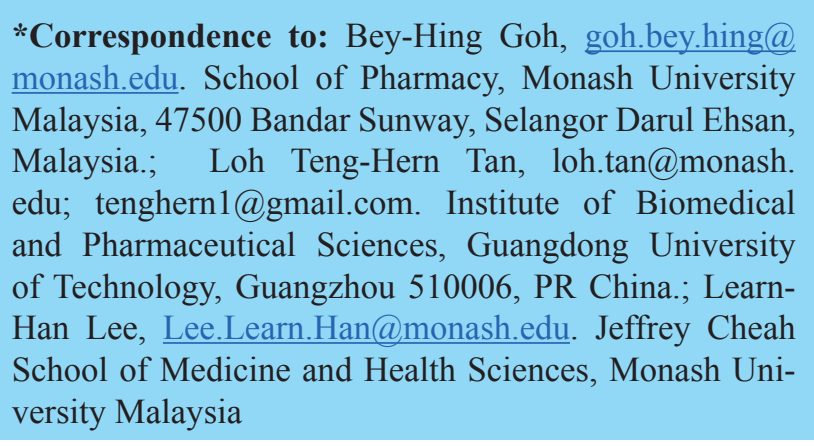

Received: $24^{\text {th }}$ April 2019

Accepted: $10^{\text {th }}$ May 2019

Published Online: $25^{\text {th }}$ May 2019

Citation: Goh JXH, Tan LTH, Yew HC, et al. Hydration effects of moisturizing gel on normal skin: A pilot study. Prog Drug Discov Biomed Sci 2019; 2(1): a0000023

\section{Introduction}

Being the outermost and largest organ of human skin serves many important functions. Conventionally, the skin is arbitrarily classified into multiple layers based on its structural distribution. The first layer, the stratum corneum, largely consists of dead cells which serves as a waterproof barrier that protects the body from mechanical injuries or invasion of harmful pathogens ${ }^{[1,2]}$. The second layer, also known as the dermis, is rich in collagen and elastin fibers and houses a large number of nerve sensors that allow detection of external stimuli such as heat, touch and pressure ${ }^{[2-4]}$. The skin plays important roles in regulating the homeostatic balance of temperature, water and electrolyte levels of the body; additionally, beneath the dermis are layers of connective tissues and adipose tissue that can serve as energy stores and also provide heat insulation $^{[2]}$.

To illustrate further, the epidermis layer can be further divided into four distinct layers, namely the stratum corneum (SC), which consist of mainly dead cells; stratum granulosum, whose name is derived from the distinct granules found after cell death; stratum spinosum, named after its spiny appearance; and finally, the deepest layer, stratum basale, where mitosis occurs and new cells are made ${ }^{[5]}$. Under normal ambience, the outermost layer, SC plays an important role in maintaining the homeostatic balance between its hydration level and the lipid layer above it to regulate the continuous function and healthy appearance of the $\operatorname{skin}^{[6]}$. While commonly known as an inert layer made up of dead cells, SC is still 
active catabolically, constantly generating water soluble compounds known as natural moisturising factor (NMF). NMF is the combination of amino acids and several other chemicals whose function is to absorb moisture for maintenance of skin hydration ${ }^{[7-9]}$.

As the underlying pathophysiology of dry skin arises from the disruption of the homeostatic balance between the skin surface lipids and moisture content in SC, maintaining skin hydration is crucial to facilitate restoration to its normal function ${ }^{[10]}$. Confronting the harsh environmental challenges, moisturizing agents render a cooling effect $^{[11]}$ and help to reduce the incidence of skin wear and tear caused by dryness ${ }^{[12]}$. There are many types of moisturizers available in the market. Depending on the nature and ingredients, different moisturizers maintain hydration through different mechanisms, such as by functioning as emollients, humectants, substantives, keratolytic agents, forming an occlusive barrier to reduce water loss, or through the combinations of several mechanisms aforementioned ${ }^{[9,13]}$.

There is a positive correlation between moisturizer use and skin hydration with numerous studies having confirmed the physiological benefits of moisturizer on skin conditions $^{[10,13,14]}$. Carville et al. showed that twice daily application of moisturizer to the older adults in an aged care facilities reduces the incidence of skin tear by almost fifty percent when compared with the controls ${ }^{[15]}$. Clearly, the use of moisturizer helps to ensure adequate hydration and prevent reduction of hydration level that may result in skin malfunction ${ }^{[16]}$.

A common natural ingredient used as moisturizer is the pulp of Aloe vera. Aloe vera is a widely used medicinal ingredient which can be dated back to more than 4000 years ago ${ }^{[17]}$. Modern research has produced evidence of its various beneficial properties such as antioxidant ${ }^{[18,19]}$, anti-inflammatory ${ }^{[20-22]}$, antibacterial ${ }^{[23]}$, hepatoprotective and immunomodulatory effects ${ }^{[24]}$, and recently even established the role of Aloe vera in counteracting ulcers ${ }^{[25]}$, diabetes $^{[26]}$ and cancers ${ }^{[27,28]}$. While it has broad application in multiple fields, the use of Aloe vera is predominant in dermatology. Aloe vera or scientifically termed Aloe barbadensis is known for its capacity to promote the healing of many skin ailments, from minor burns, cuts, frostbite, acne to more severe skin diseases and inflammation due to the fact that it can penetrate into tissues beneath the skin layers and hasten the proliferation of cells to facilitate the skin repair process ${ }^{[29,30]}$.

This study differs from many published experiments that have studied the benefits of moisturizers in the elderly and individuals with skin problems, as this experiment aims to investigate the significance of daily usage of Tested gel as a moisturizing agent in healthy young individuals, analyzing outcomes in terms of skin moisture content and skin texture improvement. As it has been proven that heat treatment in processing moisturizers will alter the polysaccharide structure, hence affecting the activity of the ge ${ }^{[31,32]}$, in this study, we also aimed to evaluate the effect of ambient storage temperature on the efficacy of Tested gel, whereby we compared storage in a refrigerator to storage at room temperature. Besides, the soothing effect rendered by the cold gel, we hypoth- esized that cooler Tested gel at $4{ }^{\circ} \mathrm{C}$ will result in a more significant rise in skin moisture content and increased smoothness of skin as compared to gel stored at room temperature (RT) of $25^{\circ} \mathrm{C}$.

\section{Methodology}

Subjects

This is a pilot study involving eight subjects. The inclusion criteria are healthy female subjects in the age range of 21 to 22 with normal skin conditions and without any previous medical history or family history of skin related diseases. The subject must have no previous experience of using the formulated gel. Informed consent was taken as the subjects agreed to commit to the 8-day trial schedule. To ensure the validity of the result, all subjects were prohibited from using other moisturizers, shampoos with moisturizing effect or any other forms of topical products during the duration of the trial.

\section{Procedures}

The subject's left ventral forearm was subdivided into three regions using a 'plastic film template' with three furrows, each with an area measurement of $3 \mathrm{~cm} \mathrm{x} 3 \mathrm{~cm}$ as illustrated in Figure 1. The first furrow (F1) is the control, no application of gel to the region. The second furrow (F2) is for the application of Tested gel stored at RT. The third furrow (F3) is for the application of the 4 ${ }^{\circ} \mathrm{C}$ Tested gel. The Tested gel used was registered under the trademark Lennisse (NOT180803966K). A thin layer of Tested gel was spread evenly over the whole exposed area in respective furrows. The $4{ }^{\circ} \mathrm{C}$ Tested gel was kept in the refrigerator before and after the application to ensure consistency of the temperature. Ethics approval from KPJ Healthcare University College was obtained (KPJUC/ RMC/SOP/EC/2018/161).

\section{Data collection}

The smoothness and moisture content of skin were the outcome measures evaluated in order to assess changes in skin conditions during the study. Although measuring body hydration levels is a rather complex process, we simplified the process by using two distinct portable devices, namely the Bioelectric Impedance Analysis (BIA) Skin Analyzer and SK-8 Digital Moisture Oil Content Analyzer. Adopting the principle of bioelectric impedance analysis, both devices are non-invasive and allow readings to be taken easily and rapidly. A standardized procedure of measurement was followed for all subjects to reduce any systemic errors.

For the BIA Skin analyzer, a slight pressure is exerted on the device against the skin so that the probe is in maximum contact with the skin surface. Readings of the moisture, oil and roughness level can be read directly from the device screen after 2 seconds. As for the SK- 8 device, the probe must be brought close to the skin and held at a perpendicular angle while measurement was taken. Oil and moisture content of the skin in percentage will appear on the LCD screen of the device after 3 seconds.

Readings were taken once from all three sites, F1, F2 and F3 on the left ventral arm at baseline ie. prior to application of investigational product $\left(\mathrm{t}_{0}\right)$, and repeated at 3 hours 
$\left(\mathrm{t}_{3}\right)$ and 6 hours $\left(\mathrm{t}_{6}\right)$ after Tested gel application respectively. The reading at site F1 acts as the negative control and the reading taken before the start of the experiment, at time zero $\left(t_{0}\right)$ is considered as the baseline value. The same process is repeated daily throughout the 8 days trial. Plastic film template with three furrows with an exact measurement of $3 \mathrm{~cm}^{2}$ was used to standardize the sites of gel application along the trial.

\section{Data Analysis}

The treatment effects of Tested gel stored under different conditions on skin hydration and smoothness were evaluated using SPSS software version 20, obtained values were expressed as mean \pm SE (standard error). For SK-8 device, the increment of the moisture content of the tested sites was calculated using the formula expressed below with slight modification ${ }^{[33]}$ :

Water content increment after $\mathrm{n}$ hours of application $(\%)=$ [(moisture percentage, $t_{n}-$ moisture percentage, $\left.t_{0}\right)$ of F2 or F3] - [(moisture percentage, $t_{n}-$ moisture percentage, $\left.t_{0}\right)$ of $\mathrm{F} 1$ region]

For BIA analyzer, the water content and smoothness scale of the tested sites were expressed as values in arbitrary units.

Arbitrary unit (AU) of moisture/texture index after $n$ hours of application of F2 or F3 = [(AU of moisture/texture index, $\mathrm{t}_{\mathrm{n}}-\mathrm{AU}$ of moisture/texture index, $\mathrm{t}_{0}$ ) of F2 or F3] [(AU of moisture/texture index, $\mathrm{t}_{\mathrm{n}}-\mathrm{AU}$ of moisture/texture index, $\mathrm{t}_{0}$ ) of $\mathrm{F} 1$ region]

Arbitrary unit (AU) of moisture/texture index after $n$ hours of application for $\mathrm{F} 1=\mathrm{AU}$ of moisture/texture index, $\mathrm{t}_{\mathrm{n}}-$ $\mathrm{AU}$ of moisture/texture index, $\mathrm{t}_{0}$ of $\mathrm{F} 1$

$\mathrm{t}_{0}=$ baseline time/time of measurements taken before gel application

Analysis of variance (ANOVA) was performed and followed by Tukey test on the differences for every parameter. Any difference is considered statistically significant if $p<$ 0.05 . Linear regression analysis was performed to evaluate the relationship between the changes in water content (\%) and days of application.

\section{Results}

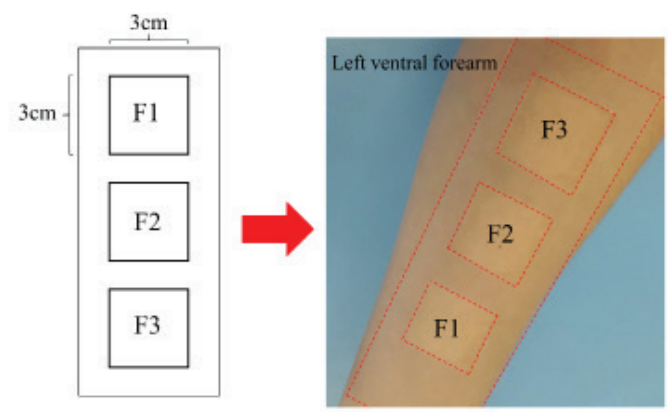

Figure 1. Plastic film template with three furrows used to standardize the sites of gel application along the trial. F1 - no gel; F2 - Tested gel stored at RT; F3 Tested gel stored at $4^{\circ} \mathrm{C}$
Tested gel exhibits immediate hydration effect on skin

Analysis of experimental results over the 8-day study period revealed that the application of Tested gel stored at both RT and $4^{\circ} \mathrm{C}$ caused significant increase $(\mathrm{p}<0.05)$ in moisture content after 3 hours of application. An estimated $6 \%$ increment of water content was recorded (by SK-8 analyzer) at both regions (F2 and F3) as compared to baseline region F1 3 hours after application of Tested gel, regardless of the storage temperature (Figure 2a). Furthermore, the improvement of moisture content was still evident at 6 hours post application, even though a slight drop in moisture content was observed as compared to the readings observed after 3 hours of application. Similarly, both devices (SK-8 analyzer and BIA analyzer) showed comparable results in which increment of water content was observed on regions applied with Tested gel at 3 and 6 hours post application. These results suggested that the Tested gel could provide immediate hydration effect on skin and was maintained up to 6 hours post application.

As opposed to the hypothesis, the Tested gel stored at $4{ }^{\circ} \mathrm{C}$ did not cause better hydration than the gel stored at RT whereby no significance difference was observed between the gels stored under either conditions. In fact, Tested gel stored at $4{ }^{\circ} \mathrm{C}$ resulted in a slightly lower increment in moisture content as compared to the effect of gel stored at RT.

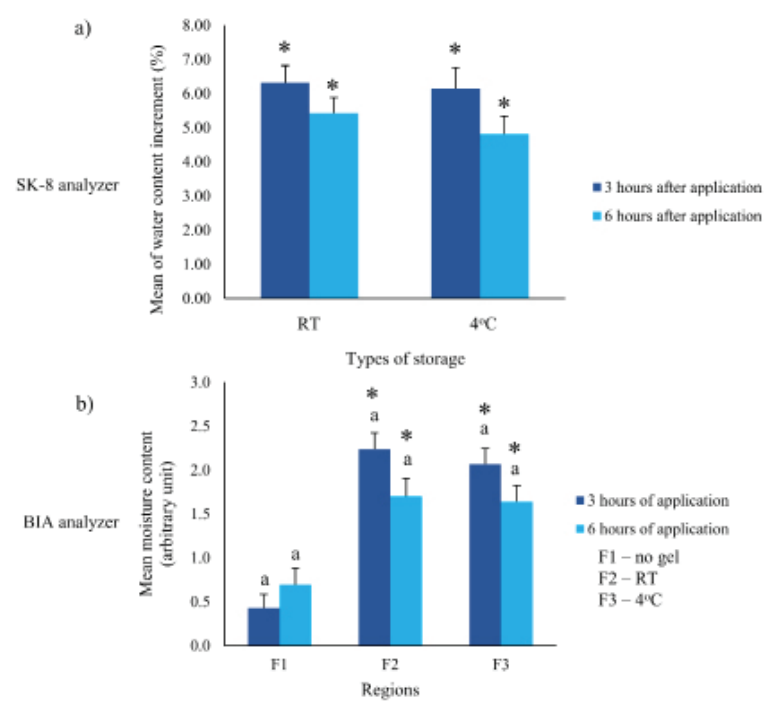

Figure 2. The immediate hydration effect of Tested gel on skin. a) The mean increment of water content was obtained by determining the difference of water content (\%) between the F2/3 regions (applied with Tested gel stored in RT or $4{ }^{\circ} \mathrm{C}$, respectively) and $\mathrm{F} 1$ region (no gel) at $\mathrm{t}_{3}$ and $\mathrm{t}_{6}$ after minus of respective baseline values recorded at $\mathrm{t}_{0}$ * indicates the significance difference between the changes in the moisture/water content of F1 region and F2/F3 regions $(\mathrm{p}<0.05)$ b) The mean increment of moisture content of each region (F1, F2 and F3) was determined by calculating the difference of the arbitrary unit between the F2/3 regions (applied with Tested gel stored in $\mathrm{RT}$ or $4{ }^{\circ} \mathrm{C}$, respectively) and $\mathrm{F} 1$ region (no gel) at $\mathrm{t}_{3}$ and $\mathrm{t}_{6}$ after minus of respective baseline arbitrary unit recorded at $\mathrm{t}_{0}$. Furrow 2 (F2): applied with gel stored at RT and Furrow 3 (F3): applied with gel stored at $4{ }^{\circ} \mathrm{C}$. Same alphabet indicates statistically insignificant difference between the mean changes in moisture content after 3 and 6 hours application, while $*$ indicates the significance difference between the changes in the moisture/water content of $\mathrm{F} 1$ region and $\mathrm{F} 2 / \mathrm{F} 3$ regions at $\mathrm{p}<0.05$. 


\section{Tested gel improves skin texture}

The skin texture is an indication of skin smoothness measured using the BIA skin analyzer. A higher texture index value implies a smoother skin texture while a lower index value indicates a rougher skin texture. At 3 hours after gel application, a significant improvement $(p<0.05)$ in texture index was noted after the use of the gels stored at both RT and at $4{ }^{\circ} \mathrm{C}$ (Figure 3 ). Moreover, the improvement in texture indexes of both $\mathrm{F} 2$ and $\mathrm{F} 3$ regions relative to baseline are still evident with significant positive values at 6 hours post application. These results suggested that the Tested gel could improve skin smoothness and the effect is maintained up to 6 hours post application

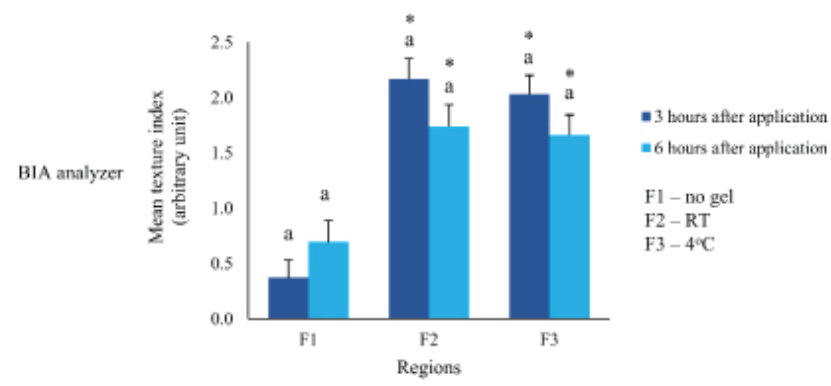

Figure 3. The skin texture improvement effect of Tested gel. The mean increment of texture index of each region (F1, F2 and F3) was determined by calculating the difference of the arbitrary unit between the F2/3 regions (applied with Tested gel stored in $\mathrm{RT}$ or $4{ }^{\circ} \mathrm{C}$, respectively) and $\mathrm{F} 1$ region (no gel) at $\mathrm{t}_{3}$ and $\mathrm{t}_{6}$ after minus of respective baseline arbitrary unit recorded at $\mathrm{t}_{0}$. Positive values of texture index indicate increment of smoothness of the skin. Furrow 2 (F2): applied with gel stored at RT and Furrow 3 (F3): applied with gel stored at $4{ }^{\circ} \mathrm{C}$. Same alphabet indicates statistically insignificant difference between the mean changes in moisture content after 3 and 6 hours application, while * indicates the significance difference between the changes in the moisture/water content of $\mathrm{F} 1$ region and $\mathrm{F} 2 / 3$ regions at $\mathrm{p}<0.05$.

\section{Cumulative hydration effect of Tested gel on skin}

The analysis of cumulative hydration effect of Tested gel on skin is shown in Figure 4. A linear regression analysis was performed to examine the changes of water content in the skin 3 hours post application of Tested gel stored at RT and $4^{\circ} \mathrm{C}$ across the 8 days trial. Based on the regression equation (Figure $4 \mathrm{a}$ ), changes in water content $(\%)$ $=4.9638+0.406$ (Days), it seems to indicate that regular

a)

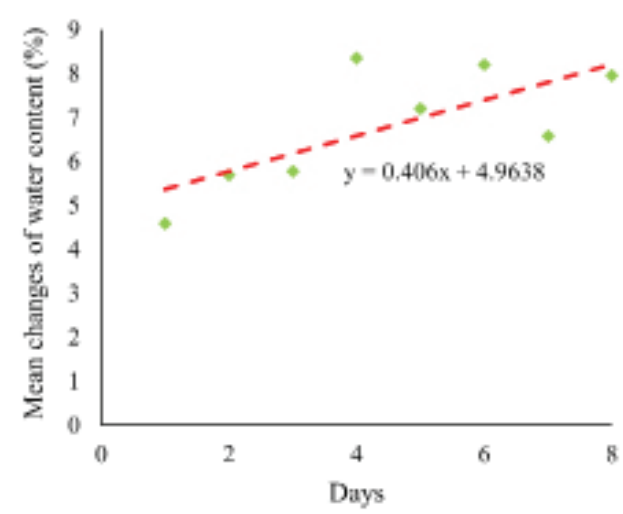

daily usage of Tested gel stored at room temperature actually boosts the skin hydration effect measured at 3 hours post application by $0.406 \%$. A similar cumulative hydration effect was also observed for the Tested gel stored at $4{ }^{\circ} \mathrm{C}$ (Figure $4 \mathrm{~b}$ ). These results demonstrated the cumulative hydration effect of Tested gel on skin with regular daily use.

\section{Discussion}

This study has proved that the use of Tested gel containing Aloe vera significantly improves the skin moisture and texture in healthy young adults. This is consistent with the study carried out by Constantin and colleagues, who discovered that after using moisturiser, the skin hydration levels in healthy subjects increased progressively over a period of one month, with an immediate $7.5 \%$ rise within one hour of application ${ }^{[34]}$. Aloe vera is one of the natural ingredients used ubiquitously as moisturizer, and its moisturizing effect is postulated to be due to its humecting effect on the skin ${ }^{[6,10,35]}$. Humectants penetrate and draw water into the $\operatorname{skin}^{[9]}$. Previous work has revealed that skin capacitance increases with prolonged use of moisturizers while the mechanical attributes remain unchanged ${ }^{[36]}$. To illustrate, a study proved that Aloe vera gel improved moisture level in SC without altering transepidermal water loss (TEWL) ${ }^{[10]}$. The moisturizing effect of Aloe vera is likely attributed to its rich polysaccharide content, such as Acemannan and Glucomannan ${ }^{[37,38]}$.

Although moisturizers are often associated with aged care, their use in young and healthy individuals is beneficial. This is due to the fact that youths are constantly exposed to various factors that may alter the BIA levels, such as physical exercise, air and humidity or hydration status, just to name a few ${ }^{[39]}$.

Findings from this study revealed that the moisturizing effect of Tested gel is cumulative over the 8 days trial, which may imply that the long-term usage of Tested gel is beneficial. The extensive work from $\mathrm{Li}$ et al. helped to confirm that single application of moisturisers with humecting effect is sufficient to act as a reliable prediction for its long-term effect ${ }^{[40]}$. This is consistent with the findings from Dal' Belo et al. who discovered the

b)

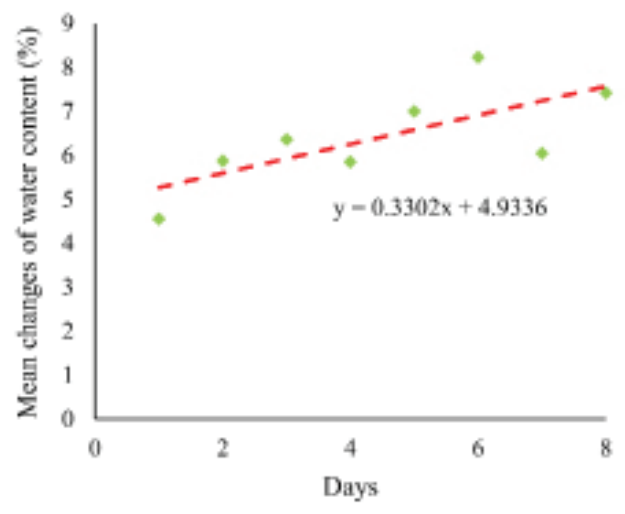

Figure 4. Linear regression analysis of the changes of water content (\%) measured at 3 hours post application along the 8 days experimental trial. a) Tested gel stored at RT. b) Tested gel stored at $4^{\circ} \mathrm{C}$. 
positive correlation between concentration of Aloe vera extract and its effectiveness ${ }^{[10]}$. Nevertheless, the long-term effect from continuous Tested gel usage needs to be further confirmed with long-term study to assess the real effects of the gel ${ }^{[41,42]}$.

\section{Study Limitations}

We compare the individual readings with baseline and normalized the values to ensure appropriateness and relevance of our findings. As aforementioned, factors such as ethnicity, environmental exposure and physical activity may affect skin quality. This study which focuses on a niche population in a tropical country may not be valid if generalized to a larger population of subjects from countries with different climates, of different occupational exposure or age difference. However, this study may hold true for both sexes, as cumulating literature showed that skin hydration level between gender is not significantly different, although female in general have a slightly higher skin hydration level in average ${ }^{[14,43]}$. Furthermore, the temperature of the gel was not measured immediately before applying with the assumption that the temperature of the gel is close to the temperature set for the fridge.

In addition to that, the complex assessment of skin health is reduced to simpler and measurable parameters in this experiment. This is achieved through the use of skin analyzing devices that function based on the theory of bioelectrical impedance analysis. Both the BIA and SK-8 analyzer are safe, non-invasive, portable and able to produce reliable and replicable results rapidly ${ }^{[44]}$. Standardized protocol is also strictly followed to reduce variables affecting the reading ${ }^{[39]}$. However, the accuracy of the testing has not been validated. Any potential adverse drug reaction is not assessed but there was no occurrence of any allergic or untoward reaction during the trial.

\section{Conclusion}

The hydrating effect of the Tested gel on skin health is indicated. Healthy young adults may benefit from the use of the Tested gel as its moisturizing effect has a measurable enhancing effect on skin hydration and smoothness. The results of the Tested gel in the improvement of skin condition are immediately evidenced following a single application with a significant increase in moisture and texture index observed at 3 hours post application. A slight decline in the hydrating effect of Tested gel was observed at 6 hours post application but a significant hydration effect was still evident. The continuous use of Tested gel may be beneficial as the result revealed a cumulative hydration related improvements in skin health induced by Tested gel. However, the long-term effect of Tested gel use has yet to be elucidated with further research. In addition, the storage temperature of Tested gel, either at RT or $4{ }^{\circ} \mathrm{C}$ does not show significant influence towards the effectiveness of Tested gel in hydrating the skin. Overall, these findings highlight the benefits of the daily usage of Tested gel in healthy young adults for moisturizing purposes to ensure adequate hydration of skin.

\section{Acknowledgement}

This project was partially funded by a research grant (KPJUC/RMC/SOP/EC/2018/161) awarded by KPJ Healthcare University College.

\section{Reference}

1. Mahendra CK, Tan LTH, Yap WH, et al. An optimized cosmetic screening assay for UVB protective property of natural products. Prog Drug Discover Biomed Sci 2019; 2(1).

2. Proksch E, Brandner JM, and Jensen JM, The skin: an indispensable barrier. Exp Dermatol 2008. 17(12): 1063-1072.

3. Poet TS and McDougal JN, Skin absorption and human risk assessment. Chem Biol Interact 2002; 140(1): 19-34.

4. Lee SH, Jeong SK, and Ahn SK, An update of the defensive barrier function of skin. Yonsei Med J 2006; 47(3): 293-306.

5. Verdier $\square$ Sévrain $\mathrm{S}$ and Bonté F, Skin hydration: A review on its molecular mechanisms. J Cosmet Dermatol 2007; 6(2): 75-82.

6. Rawlings $\mathrm{AV}$ and Harding $\mathrm{CR}$, Moisturization and skin barrier function. Dermatol Ther 2004; 17: 43-48.

7. Harding C, Long S, Richardson J, et al. The cornified cell envelope: An important marker of stratum corneum maturation in healthy and dry skin. Int J Cosmet Sci 2003; 25(4): 157-167.

8. Scott IR and Harding CR. Filaggrin breakdown to water binding compounds during development of the rat stratum corneum is controlled by the water activity of the environment. Dev Biol 1986; 115(1): 84-92.

9. Saraf S. Formulating moisturizers using natural raw materials, in Treatment of Dry Skin Syndrome. 2012; Springer. p. 379-397.

10. Dal'Belo SE, Rigo Gaspar L, and Berardo Gonçalves Maia Campos PM. Moisturizing effect of cosmetic formulations containing Aloe vera extract in different concentrations assessed by skin bioengineering techniques. Skin Res Technol 2006; 12(4): 241-246.

11. Mahor G and Ali SA. Recent update on the medicinal properties and use of Aloe vera in the treatment of various ailments. Biosci Biotechnol Res Commun 2016; 9(2): 277-292.

12. Rosado C, Pinto P, and Rodrigues LM. Assessment of moisturizer and barrier function restoration using dynamic methods. Skin Res Technol 2009; 15(1): 77-83.

13. Loden M The clinical benefit of moisturizers. J Eur Acad Dermatol Venereol; 2005 19(6): 672-688.

14. Hadi H, Awadh A, Hanif N, et al. The investigation of the skin biophysical measurements focusing on daily activities, skin care habits, and gender differences. Skin Res Technol 2016; 22(2): 247-254.

15. Carville K, Leslie G, Osseiran-Moisson R, et al. The effectivenes of a twice-daily skin-moisturising regimen for reducing the incidence of skin tears. Int Wound J 2014; 11(4): 446-453.

16. Kapoor S and Saraf S. Assessment of viscoelasticity and hydration effect of herbal moisturizers using bioengineering techniques. Pharmacogn Mag 2010; 6(24): 298.

17. Manvitha K and Bidya B. Aloe vera: A wonder plant its history, cultivation and medicinal uses. J Pharmacogn Phytochem 2014; 2(5) 85-88.

18. Kang M-C, Kim SY, Kim YT, et al. In vitro and in vivo antioxidant activities of polysaccharide purified from aloe vera (Aloe barbadensis) gel. Carbohyd Polym 2014; 99: 365-371.

19. Chun-hui L, Chang-hai W, Zhi-liang X, et al. Isolation, chemical characterization and antioxidant activities of two polysaccharides from the gel and the skin of Aloe barbadensis Miller irrigated with sea water. Process Biochem 2007; 42(6): 961-970.

20. Duansak D, Somboonwong J, and Patumraj S. Effects of Aloe vera on leukocyte adhesion and TNF- $\alpha$ and IL-6 levels in burn wounded rats. Clin Hemorheol Microcirc 2003; 29(3,4): 239-246.

21. Park M-Y, Kwon H-J, and Sung M-K. Evaluation of aloin and aloeemodin as anti-inflammatory agents in aloe by using murine macrophages. Biosci Biotechnol Biochem 2009; 73(4): 828-832.

22. Vijayalakshmi D, Dhandapani R, Jayaveni $\mathrm{S}$, et al. In vitro anti inflammatory activity of Aloe vera by down regulation of MMP9 in peripheral blood mononuclear cells. J Ethnopharmacol 2012; 141(1): $542-546$

23. Nejatzadeh-Barandozi F. Antibacterial activities and antioxidant capacity of Aloe vera. Org Med Chem Lett 2013; 3(1): 5.

24. Im S-A, Oh S-T, Song S, et al. Identification of optimal molecula size of modified Aloe polysaccharides with maximum immunomodulatory activity. Int Immunopharmacol 2005; 5(2): 271-279.

25. Cellini L, Di Bartolomeo S, Di Campli E, et al. In vitro activity of A loe vera inner gel against $\mathrm{H}$ elicobacter pylori strains. Lett Appl Microbiol 2014; 59(1): 43-48.

26. Suksomboon N, Poolsup N, and Punthanitisarn S. Effect of Aloe vera on glycaemic control in prediabetes and type 2 diabetes: A systematic review and meta-analysis. J Clin Pharm Ther 2016; 41(2): 180-188.

27. Suboj P, Babykutty S, Gopi DRV, et al. Aloe emodin inhibits colon cancer cell migration/angiogenesis by downregulating MMP-2/9, RhoB and VEGF via reduced DNA binding activity of NF- $\mathrm{kB}$. Eur J Pharm Sci 2012; 45(5): 581-591.

28. Huang P-H, Huang C-Y, Chen M-C, et al. Emodin and aloe-emodin 
suppress breast cancer cell proliferation through $\mathrm{ER} \alpha$ inhibition. Evid Based Complement Alternat Med 2013; 2013.

29. Yagi A, Egusa T, Arase M, et al. Isolation and characterization of the glycoprotein fraction with a proliferation-promoting activity on human and hamster cells in vitro from Aloe vera gel. Planta Med 1997; 63(01): $18-21$.

30. Tarameshloo M, Norouzian M, Zarein-Dolab S, et al. Aloe vera gel and thyroid hormone cream may improve wound healing in Wistar rats. Anat Cell Biol 2012; 45(3): 170-177.

31. Femenia A, García-Pascual P, Simal S, et al. Effects of heat treatment and dehydration on bioactive polysaccharide acemannan and cell wall polymers from Aloe barbadensis Miller. Carbohyd Polym 2003; 51(4): 397-405

32. Miranda M, Maureira H, Rodriguez K, et al, Influence of temperature on the drying kinetics, physicochemical properties, and antioxidant capacity of Aloe Vera (Aloe Barbadensis Miller) gel. J Food Eng 2009; 91(2): 297-304

33. Fox LT, Du Plessis J, Gerber M, et al. In Vivo skin hydration and antierythema effects of Aloe vera, Aloe ferox and Aloe marlothii gel materials after single and multiple applications. Pharmacogn Mag 2014; 10(Suppl 2): S392.

34. Constantin M-M, Poenaru E, Poenaru C, et al. Skin hydration assessment through modern non-invasive bioengineering technologies Maedica 2014; 9(1): 33 .

35. Shelton RM. Aloe vera: Its chemical and therapeutic properties. Int J Dermatol 1991; 30(10): 679-683.

36. Jemec GB and Na R. Hydration and plasticity following long-term use of a moisturizer: a single-blind study. Acta Derm Venereol 2002; $82(5)$

37. Chandegara V and Varshney A. Aloe vera L. processing and products: A review. Int J Med Aromat Plants 2013; 3: 492-506.

38. Choi S and Chung M-H. A review on the relationship between Aloe vera components and their biologic effects. Semin Integr Med 2003; 1(1): 53-62.

39. Yanovski SZ, Hubbard VS, Heymsfield SB, et al. Bioelectrical impedance analysis in body composition measurement: National Institutes of Health technology assessment conference statement. Am J Clin Nutr 1996; 64(3): 524S-532S.

40. Li F, Conroy E, Visscher M, et al. The ability of electrical measurements to predict skin moisturization. II. Correlation between one-hour measurements and long-term results. J Cosmet Sci 2001; 52(1): 23-33.

41. Berardesca E, Cosmetics EGfEMo, and Products OT. EEMCO guidance for the assessment of stratum corneum hydration: electrical methods. Skin Res Technol 1997; 3(2): 126-132.

42. De Paepe K, Derde MP, Roseeuw D, et al. Claim substantiation and efficiency of hydrating body lotions and protective creams. Contact Dermatitis 2000; 42(4): 227-234.

43. Ehlers C, Ivens U, Møller M, et al. Females have lower skin surface $\mathrm{pH}$ than men: A study on the influence of gender, forearm site variation, right/left difference and time of the day on the skin surface $\mathrm{pH}$ Skin Res Technol 2001; 7(2): 90-94.

44. Buffa R, Mereu E, Comandini O, et al. Bioelectrical impedance vector analysis (BIVA) for the assessment of two-compartment body composition. Eur J Clin Nutr 2014; 68(11): 1234. 